\title{
Orthogeriatric care pathway: a prospective survey of impact on length of stay, mortality and institutionalisation
}

\author{
N. Suhm $\cdot$ R. Kaelin $\cdot$ P. Studer $\cdot$ Q. Wang $\cdot$ \\ R. W. Kressig • D. Rikli • M. Jakob • \\ M. Pretto
}

Received: 5 March 2014/Published online: 26 July 2014

(c) Springer-Verlag Berlin Heidelberg 2014

\begin{abstract}
Introduction Care pathways for elderly hip fracture patients are increasingly implemented but there has been only limited evaluation of their use. Our objective was to investigate the impact of such a care pathway on the use of healthcare resources and on patients' outcomes.

Materials and methods The prospective survey covered 493 hip fracture patients 65 years of age or older that were treated either before "Usual Care = (UC)" or after "CoManaged-Care $=(\mathrm{CMC})$ " implementation of the care pathway. Primary outcome was length of stay (LoS). Secondary outcomes were 1 -year mortality and change in residential status from prefracture baseline to 1-year after surgery. Data were analysed by descriptive and interferential statistics and adjustment for baseline differences amongst the two patient groups was done.

Results Patients in the CMC sample had more preexisting comorbidities (CCI 2.5 versus 2.1). Prior to the fracture, a
\end{abstract}

N. Suhm and R. Kaelin are these authors have contributed equivalently to this publication.

N. Suhm $(\bowtie) \cdot$ R. Kaelin · P. Studer · D. Rikli · M. Jakob Traumatology, Department of Surgery, University Hospital Basel, Spitalstrasse 21, 4031 Basel, Switzerland

e-mail: Norbert.Suhm@usb.ch

Q. Wang

Basel Institute for Clinical Epidemiology and Biostatistics,

University Hospital Basel, Basel, Switzerland

R. W. Kressig

Felix Platter Hospital, University Center for Medicine of Aging

Basel, Basel, Switzerland

M. Pretto

Nursing Department, University Hospital Basel, Basel,

Switzerland larger proportion amongst them needed help in ADL (49 versus $26 \%$ ), and they were more likely to reside in a nursing home (36 versus $29 \%$ ). Prefracture mobility status was equal in both samples. In the CMC sample LoS was significantly shorter (LoS 8.6 versus 11.3 days, $p<0.01)$ and patients were less likely to experience a complication (59 vs $73 \%, p<0.01$ ) while being in the hospital. There was no significant difference in 1-year mortality or in change of residential status.

Conclusions A care pathway for elderly hip fracture patients allowed decreased LoS without affecting mortality or change of residential status 1 year after fracture compared to prefracture baseline.

Keywords Hip fracture program - Length of stay · Mortality · Residential status · Orthogeriatric care pathway

\section{Introduction}

The prevalence of comorbid conditions and frailty is high among elderly hip fracture patients [1-3]. They are at high risk of developing complications, of suffering from functional decline or needing long-term care, and of dying [48]. Therefore, despite successful surgical treatment, hip fractures pose a significant burden of illness for the affected patient as well as for the healthcare system [9-11].

To address this challenge a variety of orthogeriatric care models have been developed [1, 12, 13]. These models formally describe cooperation between geriatricians, orthopaedic surgeons, and other disciplines or healthcare professionals. The aim is to achieve an improvement in outcomes, and in functional recovery. In addition, a reduction of length of stay (LoS), of complications, or of readmissions is essential to assure cost effectiveness. This 
has been translated into dedicated care pathways and hip fracture programmes. They are increasingly implemented worldwide even though to date there has been only limited evaluation of their use [14]. Studies of orthogeriatric care programs have particularly been criticised for methodological weaknesses, including small sample sizes, poorly matched treatment groups, heterogeneous populations and incomplete follow-up [15]. Consequently, these studies have produced conflicting results. Another major concern is that programs that focus on reduced length of stay were reported to result in worse long-term outcomes in elderly hip fracture patients [16].

The implementation of a dedicated care pathway for older hip fracture patients at our hospital allowed us to investigate its impact on the use of healthcare resources and on patients' outcomes by means of a quality assurance survey. To our knowledge, it is the first time that such an investigation was done for large patient samples based on prospective data without exclusion of relevant patient groups. It was performed as a pre- and post-implementation survey within one institution.

\section{Patients and methods}

Description of care models under investigation

Prior to implementation of the care pathway there was no dedicated approach towards the treatment of elderly injured patients at our institution. However, a senior internal medicine resident had already been integrated into our orthotrauma team in 2004. He participated from hospital admission onwards and visited older patients daily. Furthermore, there was regular input from the physiotherapist, social worker, clinical nurse specialist and, if necessary, other professionals. This setting could best be compared with model "B" according to Pioli's classification of orthogeriatric care models $[12,13]$. This setting is named "Usual Care = (UC)" in the following sections.

Between November 2009 and March 2010 we implemented major elements of the co-managed hip fracture program as described by Friedman [17]. The patients eligible to GFC treatment enter the pathway as early as in the emergency department (Fig. 1). Furthermore, the pathway covers the operating room and the orthotrauma ward. This includes standardised orders to prevent complications which are common in elderly hip fracture patients (e.g. delirium, malnutrition, venous thrombosis, pressure sores), early participation in self-care, and protocols to foster early discharge planning. Both an orthopaedic resident and the internal medicine resident manage the patients and are responsible for surgical and medical care. By doing so, we succeeded to meet the criteria of model " $D$ " according to Pioli's classification of orthogeriatric care models. This setting is named "Co-Managed Care $=(\mathrm{CMC})$ " in the following sections.

\section{Surgical procedures}

All included patients underwent surgical correction of their hip fracture. Femoral neck fractures were treated either by hemiarthroplasty or by total hip arthroplasty with a cemented or an uncemented stem. Pertrochanteric fractures were stabilised by means of an intramedullary nail or a sliding hip screw. Decision on the specific type of surgery or implant was made based on the fracture type, on the patient's prefracture functional status, and finally on the individual surgeon's judgment.

Survey design, sample and data acquisition

All patients who were aged 65 years or older, and who were admitted for a low energy femoral neck, pertrochanteric or intertrochanteric femoral fracture to our hospital during the observation periods were included. Only patients with pathologic fractures or with fractures due to a high-energy trauma were excluded.

Patients admitted from June 1st 2007 until September 30th 2008 were treated according to the UC setting prior to implementation of the care pathway. Some results from this UC sample were reported previously [11]. Patients admitted from April 1st 2010 until March 31st 2011 were treated according to the CMC setting after implementation of the care pathway.

All data in the survey were collected prospectively as part of on-going quality improvement efforts. Data were collected by clinicians caring for the patient, including residents and surgeons, and by members of the dedicated quality assurance team. Additionally in-hospital complications were traced back by means of a retrospective chart review.

Age, gender, fracture type and pre-existing co-morbidities were retrieved from the physician's basic assessment on admission and were completed from the patient's medical record. Based on these data the index of co-morbidities was calculated as Charlson Co morbidity Index (CCI), not including age [18].

Information on prefracture mobility status, on the patient's prefracture ability to perform activities of daily living (ADL), and on prefracture residential status was obtained from the patients themselves. When patients had a diagnosis of dementia or met criteria of delirium, the information was obtained from a family member or from other caregivers. Prefracture mobility status was categorised based on the need for different walking aids as: "walking without 


\section{The Orthogeriatric Pathway}

\section{Emergency Department (Goal: Identification of patients at risk)}

- Fracture Dx: clinical and radiologic evaluation

- Preoperative assessment by anesthesist: cleared: NPO / additional evaluation required / treatment. CPR order, DNR order.

- Interdisciplinary risk assessment : delirium / malnutrition / infection / TVT

- Identification of patients eligible for GFC treatment : interdisciplinary evaluation of prefracture status (cognitive-, nutritional, functional, and mobility)

\section{Surgical intervention (Goal: Time to surgery $<24 \mathrm{~h}$ )}

- Adapted for fracture type, age and prefracture status ( see above): surgical treatment $>>$ full weight bearing as tolerated.

- Femoral neck fractures: Endoprosthesis (cemented, non-cemented), Total Hip Replacement

- Trochanteric fractures: Intramedullary Nail (short nail, long nail)

\section{Postoperative care (Goal: Limit complications)}

- Consented protocols: delirium prevention, secondary fracture prevention: Fracture Liaison Service FLS, anemia, malnutrition

- Daily interdisciplinary rounds (internal medicine resident + orthotrauma surgeon)

- Dedicated GFC rounds: scoring (Penrod, NHFS, Parker, Charlson), goal setting for rehab \& discharge planning

\section{4. discharge management (Goal: LOS $<7 d$ )}

Direct back home: scheduled follow up, FLS

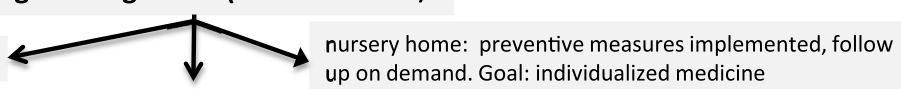

Rehabilitation center: interdisciplinary treatment. Goal: Return to prefracture residential status.

5. Follow up in outpatient clinic and FLS ( Goal: Return to prefracture status, Secondary fracture prevention implemented)

- 6 and 12 weeks postoperative clinical and radiologic Follow up

- if applicable: further osteoporosis diagnostics, treatment and scheduled follow-up within the Fracture Liaison Service (FLS) / outpatient clinic

Fig. 1 The Orthogeriatric pathway

help", "walking with stick", "walking with frame/other person", "wheelchair/bedridden", or "unknown". The patient's prefracture ability to perform ADL was categorised as "independent" (no reported need for help in any ADL), "help needed", or "unknown". The patient's residential status was classified as "home or retirement community", "nursing home", or "unknown".

The primary outcome was LoS in the acute hospital. LoS was defined as the number of days from admission to our hospital until discharge, either to the rehabilitation centre, or back to the nursing home, or directly back home.

Secondary outcomes were 1-year mortality (death within 365 days after surgery) and change in residential status from prefracture baseline until 1 year after surgery. The residential status was considered "worse" if the patient needed more help and therefore had moved to a nursing home 1 year after surgery. To minimise the number of patients that were lost to follow-up, additional information to identify patients who had died was obtained from the primary care provider or from the obituary column.

Other outcome variables were time to surgery, in-hospital complication rate, in-hospital and 30-day mortality, and 30-day and 1-year readmission rates.
Time to surgery was defined as the time (in hours) from admission into the hospital until the time when the patient arrived in the operating room.

A decision was made to validate in-hospital complications in both samples by means of an additional retrospective chart review. This task was done by one resident who was not aware of the specific sample the patients were included. Severity of complications was graded according to Dindo et al. [19] as follows: Grade 1: minor risk events/any deviation from the normal postoperative course without the need for interventions except analgesic, antipyretic, antiemetic, and antidiarrheal drugs. Grade 2: Complications requiring pharmacological treatment with drugs other than such used for grade I complications (e.g. pneumonia, delirium, lower urinary tract infection, cardiac tachyarrhythmia). Blood transfusions and total parenteral nutrition were also included. Grade 3: complication requiring surgical, endoscopic or radiological intervention. Grade 4: Life-threatening complication requiring ICU management (e.g. multiorgan dysfunction). Grade 5: Death of a patient [19].

In-hospital mortality was defined as death during initial stay in our orthotrauma unit and 30-day mortality was defined as death within 30 days after surgery, 
Table 1 Demographic data and baseline characteristics of patients

\begin{tabular}{|c|c|c|c|}
\hline $\begin{array}{l}\text { Baseline characteristic and } \\
\text { prefracture status }\end{array}$ & $\begin{array}{l}\mathrm{UC} \\
(n=269)\end{array}$ & $\begin{array}{l}\mathrm{CMC} \\
(n=224)\end{array}$ & $\begin{array}{l}\text { Total } \\
(n=493)\end{array}$ \\
\hline Age [years: mean (SD)] & $83.9(7.5)$ & $84.3(7.4)$ & $84.1(7.5)$ \\
\hline Female gender $[n(\%)]$ & $208(77)$ & $169(75)$ & $377(76)$ \\
\hline \multicolumn{4}{|l|}{ Type of fracture $[n(\%)]$} \\
\hline Formal neck & $131(49)$ & $103(46)$ & $234(47)$ \\
\hline Trochanteric & $138(51)$ & $121(54)$ & $259(53)$ \\
\hline \multicolumn{4}{|l|}{ Comorbidity } \\
\hline $\begin{array}{l}\text { Charlson Comorbidity Index } \\
\text { [mean (SD)] }\end{array}$ & $2.1(1.6)$ & $2.5(2.0)$ & $2.3(1.8)$ \\
\hline Dementia $[n(\%)]$ & $94(35)$ & $74(33)$ & $168(34)$ \\
\hline \multicolumn{4}{|l|}{ Mobility status $[n(\%)]$} \\
\hline Walking without help & $100(37)$ & $86(38)$ & $186(38)$ \\
\hline Walking with stick & $60(22)$ & 43 (19) & $103(21)$ \\
\hline $\begin{array}{l}\text { Walking with frame/other } \\
\text { person }\end{array}$ & $62(23)$ & $49(22)$ & $111(23)$ \\
\hline Wheelchair/bedridden & $9(4)$ & $8(4)$ & $17(3)$ \\
\hline Unknown & $38(14)$ & $38(17)$ & $76(15)$ \\
\hline \multicolumn{4}{|c|}{ Activities of daily living $[n(\%)]$} \\
\hline Independent & $133(50)$ & $94(42)$ & $227(46)$ \\
\hline Help needed & $71(26)$ & $109(49)$ & $180(37)$ \\
\hline Unknown & $65(24)$ & $21(9)$ & $86(17)$ \\
\hline \multicolumn{4}{|l|}{ Residential status $[n(\%)]$} \\
\hline $\begin{array}{l}\text { Home or retirement } \\
\text { community }\end{array}$ & $190(71)$ & $142(63)$ & $332(67)$ \\
\hline Nursing home or hospital & $78(29)$ & $80(36)$ & $158(32)$ \\
\hline Unknown & $1(0)$ & $2(1)$ & $3(1)$ \\
\hline
\end{tabular}

Usual Care UC sample, Co-Managed Care CMC sample

Readmission was defined as any non-elective hospital admission within the time period from the day of discharge from our orthotrauma unit until 30 days (30-day readmission rate) or until 1-year (1-year readmission rate) later. Readmissions were traced using the hospital's computerised medical records system during the relevant time periods. The 1-year follow-up telephone interview was also used to cover readmissions to external hospitals.

\section{Ethical considerations}

The survey was approved in the context of continuous quality improvement by the responsible ethical review board. Patient data were kept confidential, analysed anonymously and an accordant commitment for confidentiality was signed by all authors. In view of the frail patient population that was covered by this survey a scheduled in-
Table 2 Summary of the unadjusted outcomes

\begin{tabular}{lccc}
\hline Outcome & $\begin{array}{l}\text { UC } \\
(n=269)\end{array}$ & $\begin{array}{l}\text { CMC } \\
(n=224)\end{array}$ & $p$ value $^{\mathrm{a}}$ \\
\hline $\begin{array}{l}\text { Time to surgery [hours: mean } \\
\text { (SD)] }\end{array}$ & $27.9(24.9)$ & $30.9(28.2)$ & 0.22 \\
LoS [days: mean (SD)] & $11.3(4.8)$ & $8.6(3.9)$ & $<0.01^{*}$ \\
In-hospital complications (Clavien and Dindo & $<0.01^{*}$ \\
classification) [n (\%)] & $73(27)$ & $93(41)$ & \\
No complication & $50(19)$ & $22(10)$ & \\
Grade 1 & $113(42)$ & $96(43)$ & \\
Grade 2 & $21(8)$ & $6(3)$ & \\
Grade 3 & $6(2)$ & $3(1)$ & \\
Grade 4 & $6(2)$ & $4(2)$ & \\
Grade 5 & & & \\
Mortality [ $n(\%)]$ & $6(2)$ & $4(2)$ & 0.99 \\
In-hospital & $16(6)$ & $13(6)$ & 0.99 \\
30-day & $53(20)$ & $64(29)$ & $0.01^{*}$ \\
1-year & & & \\
Readmission [n $(\%)]$ & $9(3)$ & $15(7)$ & 0.10 \\
30-day & $85(32)$ & $64(29)$ & 0.49 \\
1-year & & & \\
\hline
\end{tabular}

* Indicates a significant difference between the Usual Care UC sample and the Co-managed Care CMC sample

a Student's $t$ test was used for continuous variables, and Fisher's exact test was used for categorical variables

person visit to obtain 30-days or 1-year follow-up data was deemed unfeasible. Instead follow-up data were obtained by telephone interviews with the patients themselves, their relatives, or the primary care providers 12 months after fracture ( \pm 2 weeks).

Statistical analysis

Demographic data and baseline characteristics are summarised for the UC sample and for the CMC sample: Table 1. We conducted crude comparisons of the outcomes between the two samples using Student's $t$ test for continuous variables and Fisher's exact test for categorical variables: Table 2 .

To address the primary outcome, LoS (days from admission to discharge) was compared between the two samples using Cox proportional hazards model. Patients were followed from the date of admission until the date of discharge or censored if they died in the hospital. We adjusted for the following confounding risk factors: age, gender, Charlson comorbidity index, residential status before the fracture, time to surgery and in-hospital complications: Table 3.

To address the secondary outcome " 1 -year mortality", time to death was analysed using Cox proportional hazards model. Patients were followed from the date of surgery 
Table 3 Time to event analyses for the primary outcome "LoS"

\begin{tabular}{lllll}
\hline Parameter & Univariate & $p$ value & Multivariate & $p$ value \\
& HR $(95 \%$ & & HR $(95 \%$ & \\
& CI) & CI $)$ & \\
\hline
\end{tabular}

\begin{tabular}{|c|c|c|c|c|}
\hline \multicolumn{5}{|l|}{ LoS } \\
\hline $\mathrm{UC}$ & Reference & - & Reference & - \\
\hline $\mathrm{CMC}$ & $\begin{array}{l}1.74(1.45, \\
2.08)\end{array}$ & $<0.01 *$ & $\begin{array}{l}1.73(1.43, \\
2.09)\end{array}$ & $<0.01^{*}$ \\
\hline $\begin{array}{l}\text { Age per year } \\
\text { increase }\end{array}$ & $\begin{array}{l}1.01(1.00, \\
1.02)\end{array}$ & 0.06 & $\begin{array}{l}1.01(1.00, \\
1.02)\end{array}$ & 0.10 \\
\hline \multicolumn{5}{|l|}{ Gender } \\
\hline Male & Reference & - & Reference & - \\
\hline Female & $\begin{array}{l}1.17(0.95, \\
1.45)\end{array}$ & 0.14 & $\begin{array}{l}1.10(0.89, \\
1.37)\end{array}$ & 0.39 \\
\hline $\begin{array}{l}\text { Charlson } \\
\text { Comorbidity } \\
\text { Index (CCI) (per } \\
\text { unit increase) }\end{array}$ & $\begin{array}{l}1.00(0.95 \\
1.05)\end{array}$ & 0.98 & $\begin{array}{l}1.00(0.95, \\
1.05)\end{array}$ & 0.92 \\
\hline \multicolumn{5}{|c|}{ Prefracture residential status } \\
\hline $\begin{array}{l}\text { Home or } \\
\text { retirement } \\
\text { community }\end{array}$ & Reference & - & Reference & - \\
\hline $\begin{array}{l}\text { Nursing home or } \\
\text { hospital }\end{array}$ & $\begin{array}{l}1.74(1.43, \\
2.11)\end{array}$ & $<0.01 *$ & $\begin{array}{l}1.93(1.57, \\
2.38)\end{array}$ & $<0.01^{*}$ \\
\hline $\begin{array}{l}\text { Time to surgery per } \\
5 \mathrm{~h} \text { increase }\end{array}$ & $\begin{array}{l}0.97(0.95 \\
0.99)\end{array}$ & $<0.01 *$ & $\begin{array}{l}0.96(0.94 \\
0.98)\end{array}$ & $<0.01^{*}$ \\
\hline \multicolumn{5}{|c|}{ In-hospital complications by Clavien and Dindo classification } \\
\hline No complication & Reference & - & Reference & - \\
\hline Grade 1 or 2 & $\begin{array}{l}0.73(0.60 \\
0.88)\end{array}$ & $<0.01 *$ & $\begin{array}{l}0.66(0.54, \\
0.81)\end{array}$ & $<0.01 *$ \\
\hline Grade $3-5$ & $\begin{array}{l}0.44(0.30 \\
0.63)\end{array}$ & $<0.01 *$ & $\begin{array}{l}0.38(0.26, \\
0.56)\end{array}$ & $<0.01^{*}$ \\
\hline
\end{tabular}

Hazards ratio $(\mathrm{HR})>1$ indicates a shorter $\mathrm{LoS}$, while $\mathrm{HR}<1$ indicates a longer LoS

CI confidence interval

* Indicates a significant difference in the LoS

until the date of death or censored when they were confirmed to be alive at 1 year after surgery or lost to followup, whichever came first. We adjusted for the same confounding risk factors as listed above: Table 4 . In a sensitivity analysis, we also adjusted for the patient's prefracture ability to perform ADL, which was an important risk factor for death but likely to have a high level of misclassification.

To address the secondary outcome "change in residential status at 1 year after surgery", a cross-sectional analysis was carried out using logistic regression models to compare the need of higher level of care at 1 year after surgery between the two samples. Consequently for this secondary outcome, we considered only the subset of patients who lived at home or in a retirement community before the fracture and who reported residential status at 1 year after surgery $(n=257)$. For the multivariate analyses, we adjusted for age, gender, prefracture mobility status and dementia documented on admission: Table 5.

All statistical analyses were done with SAS version 9.2 (SAS Institute, Inc., Cary, NC, USA).

\section{Results}

During the two recruitment periods, 501 eligible patients were admitted. Eight patients were excluded from the survey because they did not undergo surgery. Of the included patients, 269 were in the UC sample and 224 were in the CMC sample. Demographic data and baseline characteristics of patients in the two samples are given in Table 1. The CMC patients had on average higher CCI than their UC counterparts (mean CCI 2.5 versus 2.1). Furthermore, a larger proportion of patients in the CMC sample needed help in performing ADL prior to the fracture (49 versus $26 \%$ ) and they were more likely to reside in a nursing home or to be transferred from another hospital prior to the fracture (36 versus $29 \%$ ). The prefracture mobility status was similar in both samples.

Unadjusted differences in outcomes between the two samples are shown in Table 2. Patients in the CMC sample were less likely to experience a complication (59 vs $73 \%$, $p<0.01$ ) while being in the hospital. Other outcomes were similar between the two samples except for LoS and 1-year mortality.

LoS Patients in the CMC sample stayed for significantly shorter time in our orthotrauma unit: LoS 8.6 versus 11.3 days, $p<0.01$. This result was still held true when we assessed time to discharge from the hospital in a time-toevent analysis adjusted for the baseline differences between the two samples (Table 3). The sensitivity analysis where we dropped time to surgery as an independent variable led to the same conclusion.

1-year mortality Univariate analysis suggested that patients in the CMC sample experienced a significant increase of 1 -year mortality $(p=0.01)$. This significance was retained in the multivariate analysis with patients in the CMC sample having a higher risk of death within 1 year after surgery: hazards ratio (HR) 1.47 (95\% confidence interval 1.01-2.15, $p=0.05$ ). However, when prefracture ability to perform ADL was further adjusted in a sensitivity analysis, the difference in 1-year mortality between the two samples was no longer significant (HR 1.37, $95 \%$ confidence interval $0.91-2.05, \quad p=0.13$ ) (Table 4).

Residential status Among the 257 patients who lived at home or in retirement communities before the fracture, 27 out of $160(16.9 \%)$ in the UC sample and 12 out of 97 $(12.4 \%)$ in the CMC sample needed a higher level of care and moved to a nursing home within 1 year after surgery. 
Table 4 Time to event analyses for the 1-year mortality

\begin{tabular}{|c|c|c|c|c|}
\hline Parameter & Univariate HR $(95 \% \mathrm{CI})$ & $p$ value & Multivariate HR (95\% CI) & $p$ value \\
\hline \multicolumn{5}{|l|}{ 1-year mortality } \\
\hline UC & Reference & - & Reference & - \\
\hline $\mathrm{CMC}$ & $1.56(1.08,2.24)$ & $0.02 *$ & $1.47(1.01,2.15)$ & $0.05^{*}$ \\
\hline Age per year increase & $1.07(1.04,1.10)$ & $<0.01 *$ & $1.06(1.03,1.10)$ & $<0.01 *$ \\
\hline \multicolumn{5}{|l|}{ Gender } \\
\hline Male & Reference & - & Reference & - \\
\hline Female & $0.79(0.53,1.18)$ & 0.25 & $0.64(0.42,0.98)$ & $0.04 *$ \\
\hline Comorbidity (CCI) per unit increase & $1.27(1.17,1.38)$ & $<0.01 *$ & $1.23(1.12,1.35)$ & $<0.01 *$ \\
\hline \multicolumn{5}{|l|}{ Prefracture residential status } \\
\hline Home or retirement community & Reference & - & Reference & - \\
\hline Nursing homeorhospital & $3.13(2.18,4.51)$ & $<0.01 *$ & $2.13(1.46,3.11)$ & $<0.01^{*}$ \\
\hline Time to surgery per $5 \mathrm{~h}$ increase & $1.01(0.98,1.05)$ & 0.39 & $1.00(0.96,1.04)$ & 0.99 \\
\hline \multicolumn{5}{|c|}{ In-hospital complications by Clavien and Dindo classification } \\
\hline No complication & Reference & - & Reference & - \\
\hline Grade 1 and 2 & $2.15(1.35,3.42)$ & $<0.01 *$ & $1.79(1.11,2.89)$ & $0.02 *$ \\
\hline Grade $3-5$ & $3.20(1.69,6.05)$ & $<0.01^{*}$ & $2.65(1.37,5.14)$ & $<0.01 *$ \\
\hline
\end{tabular}

Hazards ratio $(\mathrm{HR})>1$ indicates a higher risk of death, while $\mathrm{HR}<1$ indicates a lower risk of death

CI confidence interval

* Indicates a significant difference in time to death within 1 year after surgery

Table 5 Cross-sectional analyses for change towards need for higher level of care 1 year after surgery

\begin{tabular}{|c|c|c|c|c|}
\hline Parameter & Univariate OR $(95 \% \mathrm{CI})$ & $p$ value & Multivariate OR (95 \% CI) & $p$ value \\
\hline \multicolumn{5}{|l|}{ Residential Status } \\
\hline $\mathrm{UC}$ & Reference & - & Reference & - \\
\hline $\mathrm{CMC}$ & $0.72(0.35,1.47)$ & 0.37 & $0.93(0.42,2.05)$ & 0.86 \\
\hline Age per year increase & $1.09(1.04,1.15)$ & $<0.01 *$ & $1.08(1.02,1.14)$ & $0.01 *$ \\
\hline \multicolumn{5}{|l|}{ Gender } \\
\hline Male & Reference & - & Reference & - \\
\hline Female & $1.60(0.67,3.82)$ & 0.29 & $1.37(0.53,3.57)$ & 0.51 \\
\hline \multicolumn{5}{|l|}{ Prefracture mobility status } \\
\hline Walking alone & Reference & - & Reference & - \\
\hline Help needed & $2.97(1.27,6.93)$ & $0.01 *$ & $1.89(0.74,4.80)$ & 0.18 \\
\hline Unknown & $3.18(1.12,9.06)$ & $0.03 *$ & $2.85(0.91,8.91)$ & 0.07 \\
\hline Dementia documented on admission & $6.37(3.04,13.35)$ & $<0.01 *$ & $5.37(2.43,11.84)$ & $<0.01^{*}$ \\
\hline
\end{tabular}

Odds ratio $(\mathrm{OR})>1$ indicates a higher probability for need of higher level of care, while $\mathrm{OR}<1$ indicates a lower probability for need of higher level of care

* Indicates a significant difference

There was no significant difference in the proportions of patients returning to their prefracture residential status between the two samples (Table 5).

\section{Discussion}

This prospective quality assurance survey shows that implementation of a care pathway for older hip fracture patients reduces length of stay and in-hospital complication rate without adversely affecting other short-term patient outcomes or the patient's long-term functional recovery. In detail there were no significant differences in short-term or long-term mortality rates, in short-term or long-term readmission rates, or in long-term change of residential status from baseline.

Despite recruitment of the two patient samples within a 4 year time frame within the same catchment area, we 
found differences in baseline characteristics between the two samples. We therefore adjusted for multiple baseline patient characteristics to determine the independent contribution from the care setting to the primary and secondary outcomes.

We decided on LoS as our primary outcome parameter because reduction thereof is an indicator of reduced utilisation of healthcare resources. Some studies, but not others, that evaluated geriatric interventions during orthopedic ward hospitalisation for hip fracture also found a reduction in the LoS [20]. The reduction of LoS in our CMC sample is merely attributable to early discharge planning.

1-year mortality rate was accepted as a secondary outcome parameter because long-term mortality rates of hip fracture patients are known from the literature. One year after hip fracture, mortality has been reported to be as high as $36 \%$ [4]. But there is also a concern when using longterm mortality as an outcome parameter in geriatric patients. Studies of causes of death based on death certificates have found that the vast majority of patients who died after a hospital admission for hip fracture died of causes not related to acute trauma [3, 21]. From this point of view long-term mortality might have only limited weight as a measure for quality of care but might better be looked upon as a parameter reporting the overall health status of the patient samples that are looked at.

The difference in 1-year mortality we found with univariate testing decreased when we applied multivariate testing. The difference became "non-significant" when baseline performance in ADL was taken into account as an additional confounding parameter. From this we conclude that 1-year mortality rates are influenced by baseline frailty.

Change of residential status from baseline until 1-year after the hip fracture was selected as the other secondary outcome parameter. It is meant to quantify long-term functional recovery. As a drawback this parameter could only be analysed in the subset of patients who really were exposed to the risk of a decline. In such patients who were already institutionalised before the fracture we could not detect a change in their level of care by means of our survey.

Other groups report that efforts to shorten LoS of hip fracture patients in acute care hospitals resulted in worse long-term outcomes [16]. Because of the lack of differences in our long-term outcome parameters we conclude that our intervention had no long-term effects in combination with the shortening of LoS.

Other studies that cover both long-term and short-term outcomes after co-managed care of geriatric hip fracture patients have yielded conflicting results. Prospective cohort studies on inpatient rehabilitation after an acute care hospital stay failed to demonstrate clinical benefits.
Randomised trials evaluated interventions similar to ours and measured outcomes beyond the time of discharge from the acute setting. Some of these studies had methodological flaws and small sample sizes. The most methodologically rigorous study which included 252 patients demonstrated slight clinical benefits only at the time of hospital discharge [22].

Only such studies with focus on in-hospital and shortterm outcomes of care merely speak in favour of the comanaged approach. The University of Rochester group described and implemented a co-managed hip-fracture program. They report reduced LoS, and fewer complications [17]. We confirmed that in-hospital complication rates were significantly lower in the CMC sample. But in our survey data on complications were collected retrospectively from the medical records. Our complication rates therefore have to be interpreted with caution.

Apart from this, the Rochester group did not find differences in short-term outcomes such as in-hospital mortality rate or 30-day readmission rate [17]. The importance of short-term mortality rates as an indicator of quality of care in geriatric hip fracture patients may be explained as follows: most excess deaths after hip fracture occur in the first 6 months. The interaction of acute injury or surgery with pre-existing co morbid conditions may contribute substantially to this early excess mortality. The capability of a specialised hip fracture program to address these interactions might therefore be measured with short-term mortality.

Readmission rates are not only looked upon as another relevant patient outcome parameter but are widely used as a measure of the quality of care and of cost-effectiveness, too. After the care pathway was implemented the shortterm (30-days) readmission rate and the long-term (1-year) readmission rate were found to be unchanged. However, readmission rates have to be considered with caution in our healthcare system. As patients have access to different hospitals, we have reliable information only on readmissions to our own hospital.

\section{Strengths and limitations}

A strength of this evaluation of a dedicated hip fracture care pathway is prospective documentation of a large and unselected sample within a defined setting. Samples of geriatric hip fracture patients tend to be inhomogeneous [8]. Strategies to overcome this issue may be patient selection or large samples. We opted for the latter approach: our sample sizes by far exceed the sample size of previous studies. Because data were gathered as part of a quality management program, analyses could be completed on all patients who met criteria for inclusion, including those with preexisting cognitive deficits. Another strength 
in this survey is the high follow-up rate of patients 1 year after the fracture, which presumes reliable results: nine patients $(3 \%)$ from the first and 14 patients $(6 \%)$ from the second sample were lost to follow-up.

Fundamental limitations of our survey design should be noted, too.

First of all our institute already showed a "culture change" among professional staff caring for older patients prior to implementation of the dedicated care pathway. Without this change in culture it is unlikely to get the benefits from the other factors, such as adoption of order sets, pathways of care, standardisation of care, etc. Comanagement programs can be seen in terms of a spectrum of care. The impact of an intervention will differ greatly based on where the institution is on the spectrum. It therefore has to be pointed out that our institution already met criteria of a hospitalist model according to Pioli's classification of orthogeriatric care models prior to implementation of the care pathway [12]. It is therefore possible that our survey underestimates the impact of the co-managed program.

Another major limitation is the lack of use of validated tools for the assessment of performance in ADL, mobility, or of cognitive impairment. Our findings are based on a quality evaluation measure for further improvement of clinical services and not a scientific study. For this reason, data was collected during routine clinical care with limited resources. The application of standard assessment tools was beyond the resources available for quality management purposes in our setting.

\section{Conclusions}

A care pathway for geriatric hip fracture patients allowed reduced LoS without adversely affecting short-term or long-term patients' outcomes.To some part our and others failure to improve outcome may also be attributed to the targeting of patients. Effective interdisciplinary geriatric interventions must target a specific population that is neither too well nor too unwell to derive benefit [23, 24]. Penrod et al. [8] examined heterogeneity in hip fracture patients in order to predict variation in functional outcomes. By using the simple prefracture characteristics of age, independence in performing ADL, and mobility status at baseline, $90 \%$ of patients could be correctly classified into groups with measurably different 6-month outcomes [8]. To demonstrate the effect of interdisciplinary geriatric interventions identification of such patients who need specific medical or multidisciplinary attention might therefore be an option for future studies.
Acknowledgements Prof. Suhm reports grants from AO Foundation, grants from Department of Surgery University Hospital Basel, during the conduct of the study; personal fees and non-financial support from Eli Lilly, from Roche, from DePuySynthes, from MSD, outside the submitted work. Dr. Friedman reports personal fees from AO North America, outside the submitted work.

Conflict of interest No conflict of interest was reported on by the other co-authors.

\section{References}

1. Friedman SM, Mendelson DA, Bingham KW, Kates SL (2009) Impact of a comanaged Geriatric Fracture Center on short-term hip fracture outcomes. Arch Intern Med 169(18):1712-1717. doi:10.1001/archinternmed.2009.321

2. Rolland Y, Abellan van Kan G, Benetos A, Blain H, Bonnefoy M, Chassagne P et al (2008) Frailty, osteoporosis and hip fracture: causes, consequences and therapeutic perspectives. J Nutr Health Aging 12(5):335-346

3. Tosteson AN, Gottlieb DJ, Radley DC, Fisher ES, Melton LJ (2007) Excess mortality following hip fracture: the role of underlying health status. Osteoporos Int 18(11):1463-1472

4. Abrahamsen B, van Staa T, Ariely R, Olson M, Cooper C (2009) Excess mortality following hip fracture: a systematic epidemiological review. Osteoporos Int. doi:10.1007/s00198-009-0920-3

5. Beck A, Gebhard F, Arand M, Krischak g, Kinzl L, Bischoff M. (2003) Die Therapie der Schenkelhalsfraktur beim alten Menschen: Komplikationen nach operativer Versorgung. Akt Traumatol;33:103-8

6. Cree M, Soskolne CL, Belseck E, Hornig J, McElhaney JE, Brant $\mathrm{R}$ et al (2000) Mortality and institutionalization following hip fracture. J Am GeriatrSoc 48(3):283-288

7. McLaughlin MA, Orosz GM, Magaziner J, Hannan EL, McGinn $\mathrm{T}$, Morrison RS et al (2006) Preoperative status and risk of complications in patients with hip fracture. J Gen Intern Med 21(3):219-225

8. Penrod JD, Litke A, Hawkes WG, Magaziner J, Koval KJ, Doucette JT et al (2007) Heterogeneity in hip fracture patients: age, functional status, and comorbidity. J Am Geriatr Soc 55(3):407-413

9. Braithwaite RS, Col NF, Wong JB (2003) Estimating hip fracture morbidity, mortality and costs. J Am GeriatrSoc 51(3):364-370

10. Bundesamt für Gesundheit (BAG)(2004). Osteoporose und Stürze im Alter. Ein Public-Health-Ansatz.Hrsg. Bundesamt für Gesundheit BAG, Bern.http://www.bag.admin.ch/shop/00048/ 00155/?lang=de. Accessed 04 March 2014

11. Pretto M, Spirig R, Kaelin R, Muri-John V, Kressig RW, Suhm N. (2010) Outcomes of elderly hip fracturepatients in the Swiss healthcare system: a survey prior to the implementation of DRGs and prior to theimplementation of a Geriatric Fracture Centre. Swiss Med Wkl 140:w13086. doi:10.4414/smw.2010.13086

12. Pioli G, Giusti A, Barone A (2008) Orthogeriatric care for the elderly with hip fractures: where are we? AgingClinExp Res 20(2):113-122

13. Kammerlander C, Roth T, Friedman SM, Suhm N, Luger TJ, Kammerlander-Knauer U et al (2010) Ortho-geriatric service-a literature review comparing different models. Osteoporos Int 21(Suppl 4):S637-S646. doi:10.1007/s00198-010-1396-x

14. Roberts HC, Pickering RM, Onslow E, Clancy M, Powell J, Roberts A et al (2004) The effectiveness of implementing a care pathway for femoral neck fracture in older people: a prospective controlled before and after study. Age Ageing 33(2):178-184 
15. Cameron ID (2005) Coordinated multidisciplinary rehabilitation after hip fracture. Disabil Rehabil 27(18-19):1081-1090

16. Fitzgerald JF, Fagan LF, Tierney WM, Dittus RS (1987) Changing patterns of hip fracture care before and after implementation of the prospective payment system. JAMA 258(2):218-221

17. Friedman SM, Mendelson DA, Kates SL, McCann RM (2008) Geriatric co-management of proximal femur fractures: total quality management and protocol-driven care result in better outcomes for a frail patient population. J Am Geriatr Soc 56(7):1349-1356. doi:10.1111/j.1532-5415.2008.01770.x

18. Sundararajan V, Henderson T, Perry C, Muggivan A, Quan H, Ghali WA (2004) New ICD-10 version of the Charlson comorbidity index predicted in-hospital mortality. J Clin Epidemiol 57(12):1288-1294 Epub 2004/12/25

19. Dindo D, Demartines N, Clavien PA (2004) Classification of surgical complications: a new proposal with evaluation in a cohort of 6336 patients and results of a survey. Ann Surg 240(2):205-213. doi:10.1097/01.sla.0000133083.54934.ae
20. Vidan M, Serra JA, Moreno C, Riquelme G, Ortiz J (2005) Efficacy of a comprehensive geriatric intervention in older patients hospitalized for hip fracture: a randomized, controlled trial. J Am Geriatr Soc 53(9):1476-1482. doi:10.1111/j.15325415.2005.53466.x

21. Gosch M, Wortz M, Nicholas JA, Doshi HK, Kammerlander C, Lechleitner M (2013) Inappropriate Prescribing as a Predictor for Long-Term Mortality after Hip Fracture. Gerontology. doi:10. $1159 / 000355327$

22. Naglie G, Tansey C, Kirkland JL, Ogilvie-Harris DJ, Detsky AS, Etchells E et al (2002) Interdisciplinary inpatient care for elderly people with hip fracture: a randomized controlled trial. CMAJ 167(1):25-32

23. Huusko TM, Karppi P, Avikainen V, Kautiainen H, Sulkava R (2000) Randomised, clinically controlled trial of intensive geriatric rehabilitation in patients with hip fracture: subgroup analysis of patients with dementia. BMJ 321(7269):1107-1111

24. Winograd CH (1991) Targeting strategies: an overview of criteria and outcomes. J Am GeriatrSoc. 39(9 Pt 2):25S-35S 